TITLE:

\title{
Weighted Triangle-Free 2-Matching Problem with Edge-Disjoint Forbidden Triangles
}

AUTHOR(S):

Kobayashi, Yusuke

\section{CITATION:}

Kobayashi, Yusuke. Weighted Triangle-Free 2-Matching Problem with Edge-Disjoint Forbidden Triangles. Integer Programming and Combinatorial Optimization 2020, 12125: 280-293

\section{ISSUE DATE:}

2020

URL:

http://hdl.handle.net/2433/252767

\section{RIGHT:}

This is a post-peer-review, pre-copyedit version of an article published in Integer Programming and Combinatorial Optimization. The final authenticated version is available online at: http://dx.doi.org/10.1007/978-3-030-45771-6_22.; The full-text file will be made open to the public on 14 April 2021 in accordance with publisher's 'Terms and Conditions for Self-Archiving', この論文は出版社版でありません。引用の際には出版社版をご確認ご利用ください。; This is not the published version. Please cite only the published version. 


\title{
Weighted Triangle-free 2-matching Problem with Edge-disjoint Forbidden Triangles ${ }^{\star}$
}

\author{
Yusuke Kobayashi ${ }^{[0000-0001-9478-7307]}$ \\ Research Institute for Mathematical Sciences, Kyoto University \\ Kyoto 606-8502, Japan \\ yusuke@kurims.kyoto-u.ac.jp
}

\begin{abstract}
The weighted $\mathcal{T}$-free 2 -matching problem is the following problem: given an undirected graph $G$, a weight function on its edge set, and a set $\mathcal{T}$ of triangles in $G$, find a maximum weight 2-matching containing no triangle in $\mathcal{T}$. When $\mathcal{T}$ is the set of all triangles in $G$, this problem is known as the weighted triangle-free 2-matching problem, which is a long-standing open problem. A main contribution of this paper is to give a first polynomial-time algorithm for the weighted $\mathcal{T}$-free 2 -matching problem under the assumption that $\mathcal{T}$ is a set of edge-disjoint triangles. In our algorithm, a key ingredient is to give an extended formulation representing the solution set, that is, we introduce new variables and represent the convex hull of the feasible solutions as a projection of another polytope in a higher dimensional space. Although our extended formulation has exponentially many inequalities, we show that the separation problem can be solved in polynomial time, which leads to a polynomial-time algorithm for the weighted $\mathcal{T}$-free 2 -matching problem.
\end{abstract}

Keywords: Triangle-free 2-matchings $\cdot b$-factors $\cdot$ Extended formulation . Polynomial-time algorithm.

\section{Introduction}

\subsection{2-matchings without Short Cycles}

In an undirected graph, an edge set $M$ is said to be a 2 -matching $y^{1}$ if each vertex is incident to at most two edges in $M$. Finding a 2-matching of maximum size is a classical combinatorial optimization problem, which can be solved efficiently by using a matching algorithm. By imposing restrictions on 2-matchings, various extensions have been introduced and studied in the literature. Among them, the problem of finding a maximum 2-matching without short cycles has attracted attentions, because it has applications to approximation algorithms for TSP and

\footnotetext{
* Supported by JSPS KAKENHI Grant Numbers JP16K16010, 16H03118, JP18H05291, and JP19H05485, Japan.

1 Although such an edge set is often called a simple 2-matching in the literature, we call it a 2-matching to simplify the description.
} 
its variants. We say that a 2 -matching $M$ is $C_{\leq k}$-free if $M$ contains no cycle of length $k$ or less, and the $C_{\leq k}$-free 2 -matching problem is to find a $C_{\leq k}$-free 2matching of maximum size in a given graph. When $k \leq 2$, every 2-matching without self-loops and parallel edges is $C_{\leq k}$-free, and hence the $C_{\leq k}$-free 2matching problem can be solved in polynomial time. On the other hand, when $n / 2 \leq k \leq n-1$, where $n$ is the number of vertices in the input graph, the $C_{\leq k}$-free 2-matching problem is NP-hard, because it decides the existence of a Hamiltonian cycle. These facts motivate us to investigate the borderline between polynomially solvable cases and NP-hard cases of the problem. Hartvigsen 12 . gave a polynomial-time algorithm for the $C_{\leq 3}$-free 2-matching problem, and Papadimitriou showed that the problem is NP-hard when $k \geq 5$ (see [6]). The polynomial solvability of the $C_{\leq 4}$-free 2 -matching problem is still open, whereas some positive results are known for special cases. For the case when the input graph is restricted to be bipartite, Hartvigsen [13, Király [18, and Frank [10] gave min-max theorems, Hartvigsen [14] and Pap [26] designed polynomial-time algorithms, Babenko [1] improved the running time, and Takazawa 28] showed decomposition theorems. Recently, Takazawa [3029] extended these results to a generalized problem. When the input graph is restricted to be subcubic, i.e., the maximum degree is at most three, Bérczi and Végh 4 gave a polynomial-time algorithm for the $C_{\leq 4}$-free 2-matching problem. Relationship between $C_{\leq k}$-free 2-matchings and jump systems is studied in [3/8]22].

There are a lot of studies also on the weighted version of the $C_{\leq k}$-free 2matching problem. In the weighted problem, an input consists of a graph and a weight function on the edge set, and the objective is to find a $C_{\leq k}$-free 2-matching of maximum total weight. Király proved that the weighted $C_{\leq 4}$-free 2-matching problem is NP-hard even if the input graph is restricted to be bipartite (see [10]), and a stronger NP-hardness result was shown in [3]. Under the assumption that the weight function satisfies a certain property called vertex-induced on every square, Makai [24] gave a polyhedral description and Takazawa [27] designed a combinatorial polynomial-time algorithm for the weighted $C_{\leq 4}$-free 2 -matching problem in bipartite graphs. The case of $k=3$, which we call the weighted triangle-free 2-matching problem, is a long-standing open problem. For the weighted triangle-free 2-matching problem in subcubic graphs, Hartvigsen and Li [15] gave a polyhedral description and a polynomial-time algorithm, followed by a slight generalized polyhedral description by Bérczi [2] and another polynomial-time algorithm by Kobayashi [19. Relationship between $C_{\leq k}$-free 2-matchings and discrete convexity is studied in [19]20]22].

\subsection{Our Results}

The previous papers on the weighted triangle-free 2-matching problem 2[15]19] deal with a generalized problem in which we are given a set $\mathcal{T}$ of forbidden triangles as an input in addition to a graph and a weight function. The objective is to find a maximum weight 2-matching that contains no triangle in $\mathcal{T}$, which we call the weighted $\mathcal{T}$-free 2-matching problem. In this paper, we focus on the case when $\mathcal{T}$ is a set of edge-disjoint triangles, i.e., no pair of triangles in $\mathcal{T}$ 
shares an edge in common. A main contribution of this paper is to give a first polynomial-time algorithm for the weighted $\mathcal{T}$-free 2-matching problem under the assumption that $\mathcal{T}$ is a set of edge-disjoint triangles. Note that we impose an assumption only on $\mathcal{T}$, and no restriction is required for the input graph. We now describe the formal statement of our result.

Let $G=(V, E)$ be an undirected graph with vertex set $V$ and edge set $E$, which might have self-loops and parallel edges. For a vertex set $X \subseteq V$, let $\delta_{G}(X)$ denote the set of edges between $X$ and $V \backslash X$. For $v \in V, \delta_{G}(\{v\})$ is simply denoted by $\delta_{G}(v)$. For $v \in V$, let $\dot{\delta}_{G}(v)$ denote the multiset of edges incident to $v \in V$, that is, a self-loop incident to $v$ is counted twice. We omit the subscript $G$ if no confusion may arise. For $b \in \mathbf{Z}_{\geq 0}^{V}$, an edge set $M \subseteq E$ is said to be a $b$-matching (resp. b-factor) if $|M \cap \dot{\delta}(v)| \leq b(v)$ (resp. $|M \cap \dot{\delta}(v)|=b(v))$ for every $v \in V$. If $b(v)=2$ for every $v \in V$, a $b$-matching and a $b$-factor are called a 2 -matching and a 2-factor, respectively. Let $\mathcal{T}$ be a set of triangles in $G$, where a triangle is a cycle of length three. For a triangle $T$, let $V(T)$ and $E(T)$ denote the vertex set and the edge set of $T$, respectively. An edge set $M \subseteq E$ is said to be $\mathcal{T}$-free if $E(T) \not \subseteq M$ for every $T \in \mathcal{T}$. For a vertex set $S \subseteq V$, let $E[S]$ denote the set of all edges with both endpoints in $S$. For an edge weight vector $w \in \mathbf{R}^{E}$, we consider the problem of finding a $\mathcal{T}$-free $b$-matching (resp. $b$-factor) maximizing $w(M)$, which we call the weighted $\mathcal{T}$-free b-matching (resp. b-factor) problem. Note that, for a set $A$ and a vector $c \in \mathbf{R}^{A}$, we denote $c(A)=\sum_{a \in A} c(a)$.

Our main result is formally stated as follows.

Theorem 1. There exists a polynomial-time algorithm for the following problem: given a graph $G=(V, E), b(v) \in \mathbf{Z}_{\geq 0}$ for each $v \in V$, a set $\mathcal{T}$ of edgedisjoint triangles, and a weight $w(e) \in \mathbf{R}$ for each $e \in E$, find $a \mathcal{T}$-free b-factor $M \subseteq E$ that maximizes the total weight $w(M)$.

A proof of this theorem is given in Section 4. Since finding a maximum weight $\mathcal{T}$-free $b$-matching can be reduced to finding a maximum weight $\mathcal{T}$-free $b$-factor by adding dummy vertices and zero-weight edges, Theorem 1 implies the following corollary.

Corollary 1. There exists a polynomial-time algorithm for the following problem: given a graph $G=(V, E), b(v) \in \mathbf{Z}_{\geq 0}$ for each $v \in V$, a set $\mathcal{T}$ of edgedisjoint triangles, and a weight $w(e) \in \mathbf{R}$ for each $e \in E$, find a $\mathcal{T}$-free $b$ matching $M \subseteq E$ that maximizes the total weight $w(M)$.

In particular, we can find a $\mathcal{T}$-free 2-matching (or 2-factor) $M \subseteq E$ that maximizes the total weight $w(M)$ in polynomial time if $\mathcal{T}$ is a set of edge-disjoint triangles.

\subsection{Key Ingredient: Extended Formulation}

A natural strategy to solve the maximum weight $\mathcal{T}$-free $b$-factor problem is to give a polyhedral description of the $\mathcal{T}$-free $b$-factor polytope as Hartvigsen and Li [15] did for the subcubic case. However, as we will see in Example 1, giving a 
system of inequalities that represents the $\mathcal{T}$-free $b$-factor polytope seems to be quite difficult even when $\mathcal{T}$ is a set of edge-disjoint triangles. A key idea of this paper is to give an extended formulation of the $\mathcal{T}$-free $b$-factor polytope, that is, we introduce new variables and represent the $\mathcal{T}$-free $b$-factor polytope as a projection of another polytope in a higher dimensional space.

Extended formulations of polytopes arising from various combinatorial optimization problems have been intensively studied in the literature, and the main focus in this area is on the number of inequalities that are required to represent the polytope. If a polytope has an extended formulation with polynomially many inequalities, then we can optimize a linear function in the original polytope by the ellipsoid method (see e.g. 11]). On the other hand, even if a linear function on a polytope can be optimized in polynomial time, the polytope does not necessarily have an extended formulation of polynomial size. In this context, the existence of a polynomial size extended formulation has attracted attentions. See survey papers [5]17] for previous work on extended formulations.

In this paper, under the assumption that $\mathcal{T}$ is a set of edge-disjoint triangles, we give an extended formulation of the $\mathcal{T}$-free $b$-factor polytope that has exponentially many inequalities (Theorem 2). In addition, we show that the separation problem for the extended formulation is solvable in polynomial time, and hence we can optimize a linear function on the $\mathcal{T}$-free $b$-factor polytope by the ellipsoid method in polynomial time. This yields a first polynomial-time algorithm for the weighted $\mathcal{T}$-free $b$-factor (or $b$-matching) problem. Note that it is rare that the first polynomial-time algorithm was designed with the aid of an extended formulation. To the best of our knowledge, the weighted linear matroid parity problem was the only such problem before this paper (see [16]).

\subsection{Organization of the Paper}

The rest of this paper is organized as follows. In Section 2, we introduce an extended formulation of the $\mathcal{T}$-free $b$-factor polytope. The outline of the correctness proof is given in Section 3. In Section 4, we give a polynomial-time algorithm for the weighted $\mathcal{T}$-factor problem and prove Theorem 1. Finally, we conclude this paper with remarks in Section 5. Some of the proofs are omitted due to the space constraint and given in the full version [21].

\section{Extended Formulation of the $\mathcal{T}$-free $\boldsymbol{b}$-factor Polytope}

Let $G=(V, E)$ be a graph, $b \in \mathbf{Z}_{>0}^{V}$ be a vector, and $\mathcal{T}$ be a set of forbidden triangles. Throughout this paper, we only consider the case when triangles in $\mathcal{T}$ are mutually edge-disjoint.

For an edge set $M \subseteq E$, define its characteristic vector $x_{M} \in \mathbf{R}^{E}$ by

$$
x_{M}(e)= \begin{cases}1 & \text { if } e \in M \\ 0 & \text { otherwise }\end{cases}
$$


The $\mathcal{T}$-free b-factor polytope is defined as $\operatorname{conv}\left\{x_{M} \mid M\right.$ is a $\mathcal{T}$-free $b$-factor in $\left.G\right\}$, where conv denotes the convex hull of vectors, and the b-factor polytope is defined similarly. Edmonds [9] shows that the $b$-factor polytope is determined by the following inequalities.

$$
\begin{array}{ll}
x(\dot{\delta}(v))=b(v) & (v \in V) \\
0 \leq x(e) \leq 1 & (e \in E) \\
\sum_{e \in F_{0}} x(e)+\sum_{e \in F_{1}}(1-x(e)) \geq 1 & \left(\left(S, F_{0}, F_{1}\right) \in \mathcal{F}\right)
\end{array}
$$

Here, $\mathcal{F}$ is the set of all triples $\left(S, F_{0}, F_{1}\right)$ such that $S \subseteq V,\left(F_{0}, F_{1}\right)$ is a partition of $\delta(S)$, and $b(S)+\left|F_{1}\right|$ is odd. Note that $x(\dot{\delta}(v))=\sum_{e \in \dot{\delta}(v)} x(e)$ and $x(e)$ is added twice if $e$ is a self-loop incident to $v$.

In order to deal with $\mathcal{T}$-free $b$-factors, we consider the following constraint in addition to (2)-(4).

$$
x(E(T)) \leq 2 \quad(T \in \mathcal{T})
$$

However, as we will see in Example1, the system of inequalities (2)-(5) does not represent the $\mathcal{T}$-free $b$-factor polytope. Note that when we consider uncapacitated 2 -factors, i.e., we are allowed to use two copies of the same edge, it is shown by Cornuejols and Pulleyblank [7] that the $\mathcal{T}$-free uncapacitated 2-factor polytope is represented by $x(e) \geq 0$ for $e \in E, x(\dot{\delta}(v))=2$ for $v \in V$, and $(5)$.

Example 1. Consider the graph $G=(V, E)$ in Figure 1. Let $b(v)=2$ for every $v \in V$ and $\mathcal{T}$ be the set of all triangles in $G$. Then, $G$ has no $\mathcal{T}$-free $b$-factor, i.e., the $\mathcal{T}$-free $b$-factor polytope is empty. For $e \in E$, let $x(e)=1$ if $e$ is drawn as a blue line in Figure 1 and let $x(e)=\frac{1}{2}$ otherwise. Then, we can easily check that $x$ satisfies (2), (3), and (5). Furthermore, since $x$ is represented as a linear combination of two $b$-factors $M_{1}$ and $M_{2}$ shown in Figures 2 and 3 , $x$ satisfies (4).

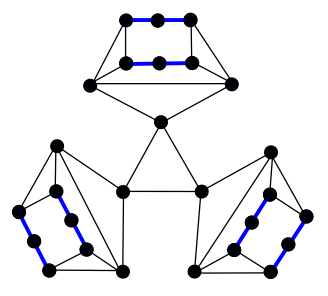

Fig. 1. Graph $G=(V, E)$

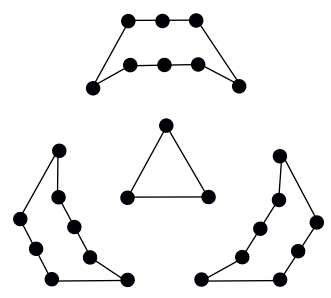

Fig. 2. $b$-factor $M_{1}$

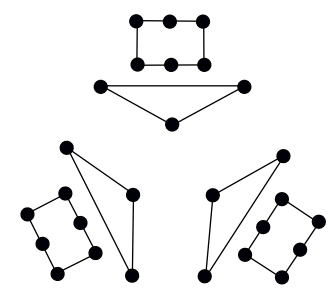

Fig. 3. $b$-factor $M_{2}$

In what follows in this section, we introduce new variables and give an extended formulation of the $\mathcal{T}$-free $b$-factor polytope. For $T \in \mathcal{T}$, we denote 
$\mathcal{E}_{T}=\{J \subseteq E(T) \mid J \neq E(T)\}$. For $T \in \mathcal{T}$ and $J \in \mathcal{E}_{T}$, we introduce a new variable $y(T, J)$. Roughly, $y(T, J)$ denotes the (fractional) amount of $b$-factors $M$ satisfying $M \cap E(T)=J$. In particular, when $x$ and $y$ are integral, $y(T, J)=1$ if and only if the $b$-factor $M$ corresponding to $(x, y)$ satisfies $M \cap E(T)=J$. We consider the following inequalities.

$$
\begin{array}{ll}
\sum_{J \in \mathcal{E}_{T}} y(T, J)=1 & (T \in \mathcal{T}) \\
\sum_{e \in J \in \mathcal{E}_{T}} y(T, J)=x(e) & (T \in \mathcal{T}, e \in E(T)) \\
y(T, J) \geq 0 & \left(T \in \mathcal{T}, J \in \mathcal{E}_{T}\right)
\end{array}
$$

If $T$ is clear from the context, $y(T, J)$ is simply denoted by $y(J)$. Since triangles in $\mathcal{T}$ are edge-disjoint, this causes no ambiguity unless $J=\emptyset$. In addition, for $\alpha, \beta \in E(T), y(\{\alpha\}), y(\{\alpha, \beta\})$, and $y(\emptyset)$ are simply denoted by $y_{\alpha}, y_{\alpha \beta}$, and $y_{\emptyset}$, respectively.

We now strengthen (4) by using $y$. For $\left(S, F_{0}, F_{1}\right) \in \mathcal{F}$, let $\mathcal{T}_{S}=\{T \in \mathcal{T}$ | $E(T) \cap \delta(S) \neq \emptyset\}$. For $T \in \mathcal{T}_{S}$ with $E(T)=\{\alpha, \beta, \gamma\}$ and $E(T) \cap \delta(S)=\{\alpha, \beta\}$, we define

$$
q^{*}(T)= \begin{cases}y_{\alpha}+y_{\alpha \gamma} & \text { if } \alpha \in F_{0} \text { and } \beta \in F_{1}, \\ y_{\beta}+y_{\beta \gamma} & \text { if } \beta \in F_{0} \text { and } \alpha \in F_{1}, \\ y_{\emptyset}+y_{\gamma} & \text { if } \alpha, \beta \in F_{1}, \\ y_{\alpha \beta} & \text { if } \alpha, \beta \in F_{0} .\end{cases}
$$

Note that this value depends on $\left(S, F_{0}, F_{1}\right) \in \mathcal{F}$ and $y$, but it is simply denoted by $q^{*}(T)$ for a notational convenience. We consider the following inequality.

$$
\sum_{e \in F_{0}} x(e)+\sum_{e \in F_{1}}(1-x(e))-\sum_{T \in \mathcal{T}_{S}} 2 q^{*}(T) \geq 1 \quad\left(\left(S, F_{0}, F_{1}\right) \in \mathcal{F}\right)
$$

For $T \in \mathcal{T}_{S}$ with $E(T)=\{\alpha, \beta, \gamma\}$ and $E(T) \cap \delta(S)=\{\alpha, \beta\}$, the contribution of $\alpha, \beta$, and $T$ to the left-hand side of $(9)$ is equal to the amount of $b$-factors $M$ such that $|M \cap\{\alpha, \beta\}| \not \equiv\left|F_{1} \cap\{\alpha, \beta\}\right|(\bmod 2)$ by the following observations.

- If $\alpha \in F_{0}$ and $\beta \in F_{1}$, then (6) and (7) show that $x(\alpha)=y_{\alpha}+y_{\alpha \beta}+y_{\alpha \gamma}$ and $1-x(\beta)=1-\left(y_{\beta}+y_{\alpha \beta}+y_{\beta \gamma}\right)=y_{\emptyset}+y_{\alpha}+y_{\gamma}+y_{\alpha \gamma}$. Therefore, $x(\alpha)+(1-x(\beta))-2 q^{*}(T)=y_{\emptyset}+y_{\gamma}+y_{\alpha \beta}$, which denotes the amount of $b$-factors $M$ such that $|M \cap\{\alpha, \beta\}|$ is even.

- If $\beta \in F_{0}$ and $\alpha \in F_{1}$, then (6) and (7) show that $(1-x(\alpha))+x(\beta)-2 q^{*}(T)=$ $y_{\emptyset}+y_{\gamma}+y_{\alpha \beta}$, which denotes the amount of $b$-factors $M$ such that $|M \cap\{\alpha, \beta\}|$ is even.

- If $\alpha, \beta \in F_{1}$, then (6) and (7) show that $(1-x(\alpha))+(1-x(\beta))-2 q^{*}(T)=$ $y_{\alpha}+y_{\beta}+y_{\alpha \gamma}+y_{\beta \gamma}$, which denotes the amount of $b$-factors $M$ such that $|M \cap\{\alpha, \beta\}|$ is odd.

- If $\alpha, \beta \in F_{0}$, then (6) and (7) show that $x(\alpha)+x(\beta)-2 q^{*}(T)=y_{\alpha}+y_{\beta}+$ $y_{\alpha \gamma}+y_{\beta \gamma}$, which denotes the amount of $b$-factors $M$ such that $|M \cap\{\alpha, \beta\}|$ is odd. 
Let $P$ be the polytope defined by

$$
P=\left\{(x, y) \in \mathbf{R}^{E} \times \mathbf{R}^{Y} \mid x \text { and } y \text { satisfy (2), (3), and (5)-(9) }\right\},
$$

where $Y=\left\{(T, F) \mid T \in \mathcal{T}, F \in \mathcal{E}_{T}\right\}$. Note that we do not need (4), because it is implied by (9). Define the projection of $P$ onto $E$ as

$$
\operatorname{proj}_{E}(P)=\left\{x \in \mathbf{R}^{E} \mid \text { There exists } y \in \mathbf{R}^{Y} \text { such that }(x, y) \in P\right\} .
$$

Our aim is to show that $\operatorname{proj}_{E}(P)$ is equal to the $\mathcal{T}$-free $b$-factor polytope. It is not difficult to see that the $\mathcal{T}$-free $b$-factor polytope is contained in $\operatorname{proj}_{E}(P)$.

Lemma 1. The $\mathcal{T}$-free b-factor polytope is contained in $\operatorname{proj}_{E}(P)$.

Proof. Suppose that $M \subseteq E$ is a $\mathcal{T}$-free $b$-factor in $G$ and define $x_{M} \in \mathbf{R}^{E}$ by 11). For $T \in \mathcal{T}$ and $J \in \mathcal{E}_{T}$, define

$$
y_{M}(T, J)= \begin{cases}1 & \text { if } M \cap E(T)=J \\ 0 & \text { otherwise }\end{cases}
$$

We can easily see that $\left(x_{M}, y_{M}\right)$ satisfies (2), (3), and (5)-(8). Thus, it suffices to show that $\left(x_{M}, y_{M}\right)$ satisfies (9). Assume to the contrary that (9) does not hold for $\left(S, F_{0}, F_{1}\right) \in \mathcal{F}$. Then, $x_{M}(e)=0$ for every $e \in F_{0} \backslash \bigcup_{T \in \mathcal{T}_{S}} E(T)$ and $x_{M}(e)=1$ for every $e \in F_{1} \backslash \bigcup_{T \in \mathcal{T}_{S}} E(T)$. Furthermore, since the contribution of $E(T) \cap \delta(S)$ and $T$ to the left-hand side of $(9)$ is equal to 1 if and only if $|M \cap E(T) \cap \delta(S)| \not \equiv\left|F_{1} \cap E(T)\right|(\bmod 2)$, we obtain $|M \cap E(T) \cap \delta(S)| \equiv$ $\left|F_{1} \cap E(T)\right|(\bmod 2)$ for every $T \in \mathcal{T}_{S}$. Then,

$$
\begin{aligned}
|M \cap \delta(S)| & =\left|(M \cap \delta(S)) \backslash \bigcup_{T \in \mathcal{T}_{S}} E(T)\right|+\sum_{T \in \mathcal{T}_{S}}|M \cap E(T) \cap \delta(S)| \\
& \equiv\left|F_{1} \backslash \bigcup_{T \in \mathcal{T}_{S}} E(T)\right|+\sum_{T \in \mathcal{T}_{S}}\left|F_{1} \cap E(T)\right|=\left|F_{1}\right| .
\end{aligned}
$$

Since $M$ is a $b$-factor, it holds that $|M \cap \delta(S)| \equiv b(S)(\bmod 2)$, which contradicts that $b(S)+\left|F_{1}\right|$ is odd.

To prove the opposite inclusion (i.e., $\operatorname{proj}_{E}(P)$ is contained in the $\mathcal{T}$-free $b$-factor polytope), we consider a relaxation of (9). For $T \in \mathcal{T}_{S}$ with $E(T)=$ $\{\alpha, \beta, \gamma\}$ and $E(T) \cap \delta(S)=\{\alpha, \beta\}$, we define

$$
q(T)= \begin{cases}y_{\alpha}+y_{\alpha \gamma} & \text { if } \alpha \in F_{0} \text { and } \beta \in F_{1}, \\ y_{\beta}+y_{\beta \gamma} & \text { if } \beta \in F_{0} \text { and } \alpha \in F_{1}, \\ y_{\gamma} & \text { if } \alpha, \beta \in F_{1}, \\ 0 & \text { if } \alpha, \beta \in F_{0} .\end{cases}
$$

Since $q(T) \leq q^{*}(T)$ for every $T \in \mathcal{T}_{S}$, the following inequality is a relaxation of (9).

$$
\sum_{e \in F_{0}} x(e)+\sum_{e \in F_{1}}(1-x(e))-\sum_{T \in \mathcal{T}_{S}} 2 q(T) \geq 1 \quad\left(\left(S, F_{0}, F_{1}\right) \in \mathcal{F}\right)
$$


Define a polytope $Q$ and its projection onto $E$ as

$$
\begin{aligned}
& Q=\left\{(x, y) \in \mathbf{R}^{E} \times \mathbf{R}^{Y} \mid x \text { and } y \text { satisfy }(2),(3),(5)-(8), \text { and }(10)\right\}, \\
& \operatorname{proj}_{E}(Q)=\left\{x \in \mathbf{R}^{E} \mid \text { There exists } y \in \mathbf{R}^{Y} \text { such that }(x, y) \in Q\right\} .
\end{aligned}
$$

Since (10) is implied by (9), we have that $P \subseteq Q$ and $\operatorname{proj}_{E}(P) \subseteq \operatorname{proj}_{E}(Q)$. In addition, we show the following proposition whose proof outline is given in Section 3

Proposition 1. $\operatorname{proj}_{E}(Q)$ is contained in the $\mathcal{T}$-free b-factor polytope.

By Lemma 1, Proposition 1, and $\operatorname{proj}_{E}(P) \subseteq \operatorname{proj}_{E}(Q)$, we obtain the following theorem.

Theorem 2. Let $G=(V, E)$ be a graph, $b(v) \in \mathbf{Z}_{\geq 0}$ for each $v \in V$, and let $\mathcal{T}$ be a set of edge-disjoint triangles. Then, both $\operatorname{proj}_{E}(P)$ and $\operatorname{proj}_{E}(Q)$ are equal to the $\mathcal{T}$-free b-factor polytope.

We remark here that we do not know how to prove directly that $\operatorname{proj}_{E}(P)$ is contained in the $\mathcal{T}$-free $b$-factor polytope. Introducing $\operatorname{proj}_{E}(Q)$ and considering Proposition 1, which is a stronger statement, is a key idea in our proof. We also note that our algorithm in Section 4 is based on the fact that the $\mathcal{T}$-free $b$-factor polytope is equal to $\operatorname{proj}_{E}(P)$. In this sense, both $\operatorname{proj}_{E}(P)$ and $\operatorname{proj}_{E}(Q)$ play important roles in this paper.

Example 2. Suppose that $G=(V, E), b \in \mathbf{Z}_{\geq 0}^{V}$, and $x \in \mathbf{R}^{E}$ are as in Example 1 . Let $T$ be the central triangle in $G$ and let $E(T)=\{\alpha, \beta, \gamma\}$. If $y \in \mathbf{R}^{Y}$ satisfies (6) and (8), then $y_{\alpha \beta}+y_{\beta \gamma}+y_{\alpha \gamma} \leq 1$. Thus, without loss of generality, we may assume that $y_{\alpha \beta} \leq \frac{1}{3}$ by symmetry. Let $S$ be a vertex set with $\delta(S)=\{\alpha, \beta\}$. Then, 10. does not hold for $(S,\{\alpha\},\{\beta\}) \in \mathcal{F}$, because $x(\alpha)+(1-x(\beta))-2 q(T)=$ $1-x(\alpha)-x(\beta)+2 y_{\alpha \beta} \leq \frac{2}{3}<1$. Therefore, $x$ is not in $\operatorname{proj}_{E}(Q)$.

\section{Outline of the Proof of Proposition 1}

In this section, we describe the outline of the proof of Proof of Proposition 1. In our proof, we use the following lemma whose proof is given in Appendix A

Lemma 2. Let $x$ be an extreme point of $\operatorname{proj}_{E}(Q)$. Then, one of the following holds.

(i) $x=x_{M}$ for some $\mathcal{T}$-free $b$-factor $M \subseteq E$.

(ii) (5) is tight for some $T \in \mathcal{T}$.

(iii) There exists a vector $y \in \mathbf{R}^{Y}$ with $(x, y) \in Q$ such that (10) is tight for some $\left(S, F_{0}, F_{1}\right) \in \mathcal{F}$ with $\mathcal{T}_{S}^{+} \neq \emptyset$, where we define $\mathcal{T}_{S}^{+}=\{T \in \mathcal{T} \mid E(T) \cap \delta(S) \cap$ $\left.F_{1} \neq \emptyset\right\}$. 
We prove Proposition 1 by induction on $|\mathcal{T}|$. If $|\mathcal{T}|=0$, then $y$ does not exist and $(10)$ is equivalent to (4). Thus, $\operatorname{proj}_{E}(Q)$ is the $b$-factor polytope, which shows the base case of the induction.

Fix an instance $(G, b, \mathcal{T})$ with $|\mathcal{T}| \geq 1$ and assume that Proposition 1 holds for instances with smaller $|\mathcal{T}|$. Suppose that $Q \neq \emptyset$, which implies that $b(V)$ is even as $(V, \emptyset, \emptyset) \notin \mathcal{F}$ by $(10)$. Pick up an extreme point $x$ of $\operatorname{proj}_{E}(Q)$ and let $y \in \mathbf{R}^{Y}$ be a vector with $(x, y) \in Q$. Our aim is to show that $x$ is contained in the $\mathcal{T}$-free $b$-factor polytope.

We apply Lemma 2 to obtain one of (i), (ii), and (iii). If (i) holds, that is, $x=x_{M}$ for some $\mathcal{T}$-free $b$-factor $M \subseteq E$, then $x$ is obviously in the $\mathcal{T}$-free $b$-factor polytope. If (ii) holds, that is, (5) is tight for some $T \in \mathcal{T}$, then we replace $T$ with a certain graph and apply the induction. If (iii) holds, that is, 10 is tight for some $\left(S, F_{0}, F_{1}\right) \in \mathcal{F}$ with $\mathcal{T}_{S}^{+} \neq \emptyset$, then we divide $G$ into two graphs, where one corresponds to $S$ and the other corresponds to $V \backslash S$, apply the induction for each graph, and merge them. See the full version [21] for a complete proof.

\section{Algorithm}

In this section, we give a polynomial-time algorithm for the weighted $\mathcal{T}$-free $b$-factor problem and prove Theorem 1. Our algorithm is based on the ellipsoid method using the fact that the $\mathcal{T}$-free $b$-factor polytope is equal to $\operatorname{proj}_{E}(P)$ (Theorem 2). In order to apply the ellipsoid method, we need a polynomial-time algorithm for the separation problem. That is, for $(x, y) \in \mathbf{R}^{E} \times \mathbf{R}^{Y}$, we need a polynomial-time algorithm that concludes $(x, y) \in P$ or returns a violated inequality.

Let $(x, y) \in \mathbf{R}^{E} \times \mathbf{R}^{Y}$. We can easily check whether $(x, y)$ satisfies (2), (3), and (5)- (8) or not in polynomial time. In order to solve the separation problem for $(9)$, we use the following theorem, which implies that the separation problem for (4) can be solved in polynomial time.

Theorem 3 (Padberg-Rao 25] (see also [23])). Suppose we are given a graph $G^{\prime}=\left(V^{\prime}, E^{\prime}\right), b^{\prime} \in \mathbf{Z}_{>0}^{V^{\prime}}$, and $x^{\prime} \in[0,1]^{E^{\prime}}$. Then, in polynomial time, we can compute $S^{\prime} \subseteq V^{\prime}$ and a partition $\left(F_{0}^{\prime}, F_{1}^{\prime}\right)$ of $\delta_{G^{\prime}}\left(S^{\prime}\right)$ that minimize $\sum_{e \in F_{0}^{\prime}} x^{\prime}(e)+\sum_{e \in F_{1}^{\prime}}\left(1-x^{\prime}(e)\right)$ subject to $b^{\prime}\left(S^{\prime}\right)+\left|F_{1}^{\prime}\right|$ is odd.

In what follows, we reduce the separation problem for $(9)$ to that for (4) and utilize Theorem 3. Suppose that $(x, y) \in \mathbf{R}^{E} \times \mathbf{R}^{Y}$ satisfies (2), (3), and (5)-(8). For each triangle $T \in \mathcal{T}$, we remove $E(T)$ and add a vertex $r_{T}$ together with three new edges $e_{1}=r_{T} v_{1}, e_{2}=r_{T} v_{2}$, and $e_{3}=r_{T} v_{3}$ (Figure 4). Let $E_{T}^{\prime}=\left\{e_{1}, e_{2}, e_{3}\right\}$ and define $x^{\prime}\left(e_{1}\right)=x(\alpha)+x(\gamma)-2 y_{\alpha \gamma}, x^{\prime}\left(e_{2}\right)=x(\alpha)+x(\beta)-2 y_{\alpha \beta}$, and $x^{\prime}\left(e_{3}\right)=x(\beta)+x(\gamma)-2 y_{\beta \gamma}$. Let $G^{\prime}=\left(V^{\prime}, E^{\prime}\right)$ be the graph obtained from $G$ by applying this procedure for every $T \in \mathcal{T}$. Define $b^{\prime} \in \mathbf{Z}_{>0}^{V^{\prime}}$ as $b^{\prime}(v)=b(v)$ for $v \in V$ and $b^{\prime}(v)=0$ for $v \in V^{\prime} \backslash V$. By setting $x^{\prime}(e)=x(\bar{e})$ for $e \in E^{\prime} \cap E$ and by defining $x^{\prime}(e)$ as above for $e \in E^{\prime} \backslash E$, we obtain $x^{\prime} \in[0,1]^{E^{\prime}}$. Then, we can show the following lemma whose proof is given in Appendix $B$. 


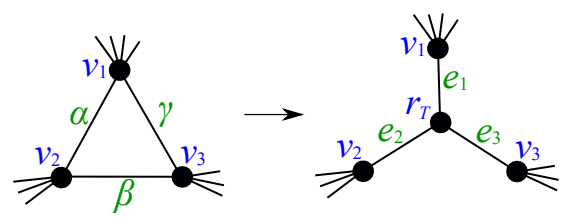

Fig. 4. Replacement of a triangle $T \in \mathcal{T}$

Lemma 3. Suppose that $(x, y) \in \mathbf{R}^{E} \times \mathbf{R}^{Y}$ satisfies (2), (3), and (5)-(8). Define $G^{\prime}=\left(V^{\prime}, E^{\prime}\right), b^{\prime}$, and $x^{\prime}$ as above. Then, $(x, y)$ violates 91 for some $\left(S, F_{0}, F_{1}\right) \in$ $\mathcal{F}$ if and only if there exist $S^{\prime} \subseteq V^{\prime}$ and a partition $\left(F_{0}^{\prime}, F_{1}^{\prime}\right)$ of $\delta_{G^{\prime}}\left(S^{\prime}\right)$ such that $b^{\prime}\left(S^{\prime}\right)+\left|F_{1}^{\prime}\right|$ is odd and $\sum_{e \in F_{0}^{\prime}} x^{\prime}(e)+\sum_{e \in F_{1}^{\prime}}\left(1-x^{\prime}(e)\right)<1$.

Since our proof of Lemma 3 is constructive, given $S^{\prime} \subseteq V^{\prime}$ and $F_{0}^{\prime}, F_{1}^{\prime} \subseteq E^{\prime}$ such that $\left(F_{0}^{\prime}, F_{1}^{\prime}\right)$ is a partition of $\delta_{G^{\prime}}\left(S^{\prime}\right), b^{\prime}\left(S^{\prime}\right)+\left|F_{1}^{\prime}\right|$ is odd, and $\sum_{e \in F_{0}^{\prime}} x^{\prime}(e)+$ $\sum_{e \in F_{1}^{\prime}}\left(1-x^{\prime}(e)\right)<1$, we can construct $\left(S, F_{0}, F_{1}\right) \in \mathcal{F}$ for which $(x, y)$ violates (9) in polynomial time. By combining this with Theorem 3 , it holds that the separation problem for $P$ can be solved in polynomial time. Therefore, the ellipsoid method can maximize a linear function on $P$ in polynomial time (see e.g. [11]), and hence we can maximize $\sum_{e \in E} w(e) x(e)$ subject to $x \in \operatorname{proj}_{E}(P)$. By perturbing the objective function if necessary, we can obtain a maximizer $x^{*}$ that is an extreme point of $\operatorname{proj}_{E}(P)$. Since each extreme point of $\operatorname{proj}_{E}(P)$ corresponds to a $\mathcal{T}$-free $b$-factor by Theorem $2 x^{*}$ is a characteristic vector of a maximum weight $\mathcal{T}$-free $b$-factor. This completes the proof of Theorem 1 .

\section{Concluding Remarks}

This paper gives a first polynomial-time algorithm for the weighted $\mathcal{T}$-free $b$ matching problem where $\mathcal{T}$ is a set of edge-disjoint triangles. A key ingredient is an extended formulation of the $\mathcal{T}$-free $b$-factor polytope with exponentially many inequalities. As we mentioned in Section 1.3 , it is rare that the first polynomialtime algorithm was designed with the aid of an extended formulation. This approach has a potential to be used for other combinatorial optimization problems for which no polynomial-time algorithm is known.

Some interesting problems remain open. Since the algorithm proposed in this paper relies on the ellipsoid method, it is natural to ask whether we can design a combinatorial polynomial-time algorithm. It is also open whether our approach can be applied to the weighted $C_{\leq 4}$-free $b$-matching problem in general graphs under the assumption that the forbidden cycles are edge-disjoint and the weight is vertex-induced on every square. In addition, the weighted $C_{\leq 3}$-free 2matching problem and the $C_{\leq 4}$-free 2 -matching problem are big open problems in this area. 


\section{References}

1. Maxim A. Babenko. Improved algorithms for even factors and square-free simple $b-$ matchings. Algorithmica, 64(3):362-383, 2012. doi:10.1007/s00453-012-9642-6.

2. Kristóf Bérczi. The triangle-free 2-matching polytope of subcubic graphs. Technical Report TR-2012-2, Egerváry Research Group, 2012.

3. Kristóf Bérczi and Yusuke Kobayashi. An algorithm for $(n-3)$-connectivity augmentation problem: Jump system approach. Journal of Combinatorial Theory, Series B, 102(3):565-587, 2012. doi:10.1016/j.jctb.2011.08.007

4. Kristóf Bérczi and László A. Végh. Restricted $b$-matchings in degree-bounded graphs. In Integer Programming and Combinatorial Optimization, pages 43-56. Springer Berlin Heidelberg, 2010. doi:10.1007/978-3-642-13036-6_4.

5. Michele Conforti, Gérard Cornuéjols, and Giacomo Zambelli. Extended formulations in combinatorial optimization. 4OR, 8(1):1-48, 2010. doi:10.1007/ s10288-010-0122-z.

6. Gérard Cornuéjols and William Pulleyblank. A matching problem with side conditions. Discrete Mathematics, 29(2):135-159, 1980. doi:10.1016/0012-365x(80) 90002-3.

7. Gérard Cornuejols and William R. Pulleyblank. Perfect triangle-free 2-matchings. In Mathematical Programming Studies, pages 1-7. Springer Berlin Heidelberg, 1980. doi:10.1007/bfb0120901

8. William H. Cunningham. Matching, matroids, and extensions. Mathematical Programming, 91(3):515-542, 2002. doi:10.1007/s101070100256

9. Jack Edmonds. Maximum matching and a polyhedron with 0,1-vertices. Journal of Research of the National Bureau of Standards B, 69:125-130, 1965.

10. András Frank. Restricted t-matchings in bipartite graphs. Discrete Applied Mathematics, 131(2):337-346, 2003. doi:10.1016/s0166-218x(02)00461-4.

11. Martin Grötschel, Lászlo Lovász, and Alexander Schrijver. Geometric Algorithms and Combinatorial Optimization, volume 2 of Algorithms and Combinatorics. Springer, 1988.

12. David Hartvigsen. Extensions of Matching Theory. PhD thesis, Carnegie Mellon University, 1984.

13. David Hartvigsen. The square-free 2-factor problem in bipartite graphs. In Integer Programming and Combinatorial Optimization, pages 234-241. Springer Berlin Heidelberg, 1999. doi:10.1007/3-540-48777-8_18.

14. David Hartvigsen. Finding maximum square-free 2-matchings in bipartite graphs. Journal of Combinatorial Theory, Series B, 96(5):693-705, 2006. doi:10.1016/j . jctb.2006.01.004.

15. David Hartvigsen and Yanjun Li. Polyhedron of triangle-free simple 2-matchings in subcubic graphs. Mathematical Programming, 138(1-2):43-82, 2012. doi:10. 1007/s10107-012-0516-0.

16. Satoru Iwata and Yusuke Kobayashi. A weighted linear matroid parity algorithm. In Proceedings of the 49th Annual ACM SIGACT Symposium on Theory of Computing - STOC 2017, pages 264-276. ACM Press, 2017. doi:10.1145/3055399. 3055436

17. Volker Kaibel. Extended formulations in combinatorial optimization. Technical report, arXiv:1104.1023, 2011.

18. Zoltán Király. $C_{4}$-free 2-factors in bipartite graphs. Technical Report TR-2012-2, Egerváry Research Group, 1999. 
19. Yusuke Kobayashi. A simple algorithm for finding a maximum triangle-free 2matching in subcubic graphs. Discrete Optimization, 7(4):197-202, 2010. doi: 10.1016/j.disopt.2010.04.001.

20. Yusuke Kobayashi. Triangle-free 2-matchings and M-concave functions on jump systems. Discrete Applied Mathematics, 175:35-42, 2014. doi:10.1016/j.dam. 2014.05 .016

21. Yusuke Kobayashi. Weighted triangle-free 2-matching problem with edge-disjoint forbidden triangles. arXiv:1911.06436, 2019.

22. Yusuke Kobayashi, Jácint Szabó, and Kenjiro Takazawa. A proof of Cunningham's conjecture on restricted subgraphs and jump systems. Journal of Combinatorial Theory, Series B, 102(4):948-966, 2012. doi:10.1016/j.jctb.2012.03.003.

23. Adam N. Letchford, Gerhard Reinelt, and Dirk Oliver Theis. Odd minimum cut sets and $b$-matchings revisited. SIAM Journal on Discrete Mathematics, 22(4):1480-1487, 2008. doi:10.1137/060664793.

24. Márton Makai. On maximum cost $K_{t, t}$-free $t$-matchings of bipartite graphs. SIAM Journal on Discrete Mathematics, 21(2):349-360, 2007. doi:10.1137/060652282.

25. Manfred W. Padberg and M. R. Rao. Odd minimum cut-sets and $b$-matchings. Mathematics of Operations Research, 7(1):67-80, 1982.

26. Gyula Pap. Combinatorial algorithms for matchings, even factors and squarefree 2-factors. Mathematical Programming, 110(1):57-69, 2007. doi:10.1007/ s10107-006-0053-9.

27. Kenjiro Takazawa. A weighted $K_{t, t}$-free $t$-factor algorithm for bipartite graphs. Mathematics of Operations Research, 34(2):351-362, 2009. doi:10.1287/moor. 1080.0365

28. Kenjiro Takazawa. Decomposition theorems for square-free 2-matchings in bipartite graphs. Discrete Applied Mathematics, 233:215-223, 2017. doi:10.1016/j. dam.2017.07.035

29. Kenjiro Takazawa. Excluded $t$-factors in bipartite graphs: A unified framework for nonbipartite matchings and restricted 2-matchings. In Integer Programming and Combinatorial Optimization, pages 430-441. Springer International Publishing, 2017. doi:10.1007/978-3-319-59250-3_35

30. Kenjiro Takazawa. Finding a maximum 2-matching excluding prescribed cycles in bipartite graphs. Discrete Optimization, 26:26-40, 2017. doi:10.1016/j.disopt. 2017.05 .003 


\section{A Proof of Lemma 2}

In this section, we give a proof of Lemma 2, We begin with the following easy lemma.

Lemma 4. Suppose that $x \in \mathbf{R}^{E}$ satisfies (3) and (5). Then, there exists $y \in$ $\mathbf{R}^{Y}$ that satisfies (6) $-(8)$.

Proof. Let $T \in \mathcal{T}$ be a triangle with $E(T)=\{\alpha, \beta, \gamma\}$ and $x(\alpha) \geq x(\beta) \geq x(\gamma)$. For $J \in \mathcal{E}_{T}$, we define $y(T, J)$ as follows.

- If $x(\alpha) \geq x(\beta)+x(\gamma)$, then $y_{\alpha \beta}=x(\beta), y_{\alpha \gamma}=x(\gamma), y_{\emptyset}=1-x(\alpha), y_{\alpha}=$ $x(\alpha)-x(\beta)-x(\gamma)$, and $y_{\beta}=y_{\gamma}=y_{\beta \gamma}=0$.

- If $x(\alpha)<x(\beta)+x(\gamma)$, then $y_{\alpha \beta}=\frac{1}{2}(x(\alpha)+x(\beta)-x(\gamma)), y_{\alpha \gamma}=\frac{1}{2}(x(\alpha)+$ $x(\gamma)-x(\beta)), y_{\beta \gamma}=\frac{1}{2}(x(\beta)+x(\gamma)-x(\alpha)), y_{\emptyset}=1-\frac{1}{2}(x(\alpha)+x(\beta)+x(\gamma))$, and $y_{\alpha}=y_{\beta}=y_{\gamma}=0$.

Then, $y$ satisfies (6)-8).

By using this lemma, we can prove Lemma 2

Proof (Proof of Lemma 2). We prove (i) by assuming that (ii) and (iii) do not hold. Since 10 is not tight for any $\left(S, F_{0}, F_{1}\right) \in \mathcal{F}$ with $\mathcal{T}_{S}^{+} \neq \emptyset, x$ is an extreme point of

$$
\left\{x \in \mathbf{R}^{E} \mid \text { There exists } y \in \mathbf{R}^{Y} \text { such that }(x, y) \text { satisfies }[2]-80\right\},
$$

because (4) is a special case of 100 in which $\mathcal{T}_{S}^{+}=\emptyset$. By Lemma 4 , this polytope is equal to $\left\{x \in \mathbf{R}^{E} \mid x\right.$ satisfies (2)-(5) $\}$. Since (5) is not tight for any $T \in \mathcal{T}$, $x$ is an extreme point of $\left\{x \in \mathbf{R}^{E} \mid x\right.$ satisfies (2)-(4) $\}$, which is the $b$-factor polytope. Thus, $x$ is a characteristic vector of a $b$-factor. Since $x$ satisfies (5), it holds that $x=x_{M}$ for some $\mathcal{T}$-free $b$-factor $M \subseteq E$.

\section{B Proof of Lemma 3}

In this section, we give a proof of Lemma 3

First, to show the "only if" part, assume that $(x, y)$ violates $(9)$ for some $\left(S, F_{0}, F_{1}\right) \in \mathcal{F}$. Recall that $\mathcal{T}_{S}=\left\{T \in \mathcal{T} \mid E(T) \cap \delta_{G}(S) \neq \emptyset\right\}$. Define $S^{\prime} \subseteq V^{\prime}$ by $S^{\prime}=S \cup\left\{r_{T}|T \in \mathcal{T}| V,(T) \cap S \mid \geq 2\right\}$. Then, for each $T \in \mathcal{T}_{S}, E_{T}^{\prime} \cap \delta_{G^{\prime}}\left(S^{\prime}\right)$ consists of a single edge, which we denote $e_{T}$. Define $F_{0}^{\prime}$ and $F_{1}^{\prime}$ as follows:

$$
\begin{aligned}
& F_{0}^{\prime}=\left(F_{0} \cap E^{\prime}\right) \cup\left\{e_{T}\left|T \in \mathcal{T}_{S},\right| E(T) \cap F_{1} \mid=0 \text { or } 2\right\}, \\
& F_{1}^{\prime}=\left(F_{1} \cap E^{\prime}\right) \cup\left\{e_{T}\left|T \in \mathcal{T}_{S},\right| E(T) \cap F_{1} \mid=1\right\} .
\end{aligned}
$$

It is obvious that $\left(F_{0}^{\prime}, F_{1}^{\prime}\right)$ is a partition of $\delta_{G^{\prime}}\left(S^{\prime}\right)$ and $b^{\prime}\left(S^{\prime}\right)+\left|F_{1}^{\prime}\right| \equiv b(S)+\left|F_{1}\right| \equiv$ $1(\bmod 2)$.

To show that $\sum_{e \in F_{0}^{\prime}} x^{\prime}(e)+\sum_{e \in F_{1}^{\prime}}\left(1-x^{\prime}(e)\right)<1$, we evaluate $x^{\prime}\left(e_{T}\right)$ or $1-x^{\prime}\left(e_{T}\right)$ for each $T \in \mathcal{T}_{S}$. Let $T \in \mathcal{T}_{S}$ be a triangle such that $E(T)=\{\alpha, \beta, \gamma\}$ and $E(T) \cap \delta_{G}(S)=\{\alpha, \beta\}$. Then, we obtain the following by the definition of $q^{*}(T)$. 
- If $T \in \mathcal{T}_{S}$ and $\alpha, \beta \in F_{0}$, then $x(\alpha)+x(\beta)-2 q^{*}(T)=x^{\prime}\left(e_{T}\right)$.

- If $T \in \mathcal{T}_{S}$ and $\alpha, \beta \in F_{1}$, then $(1-x(\alpha))+(1-x(\beta))-2 q^{*}(T)=x^{\prime}\left(e_{T}\right)$.

- If $T \in \mathcal{T}_{S}, \alpha \in F_{0}$, and $\beta \in F_{1}$, then $x(\alpha)+(1-x(\beta))-2 q^{*}(T)=y_{\emptyset}+y_{\gamma}+$ $y_{\alpha \beta}=1-x^{\prime}\left(e_{T}\right)$.

- If $T \in \mathcal{T}_{S}, \beta \in F_{0}$, and $\alpha \in F_{1}$, then $(1-x(\alpha))+x(\beta)-2 q^{*}(T)=y_{\emptyset}+y_{\gamma}+$ $y_{\alpha \beta}=1-x^{\prime}\left(e_{T}\right)$.

With these observations, we obtain

$$
\sum_{e \in F_{0}^{\prime}} x^{\prime}(e)+\sum_{e \in F_{1}^{\prime}}\left(1-x^{\prime}(e)\right)=\sum_{e \in F_{0}} x(e)+\sum_{e \in F_{1}}(1-x(e))-\sum_{T \in \mathcal{T}_{S}} 2 q^{*}(T)<1,
$$

which shows the "only if" part.

We next show the "if" part. For edge sets $F_{0}^{\prime}, F_{1}^{\prime} \subseteq E^{\prime}$, we denote $g\left(F_{0}^{\prime}, F_{1}^{\prime}\right)=$ $\sum_{e \in F_{0}^{\prime}} x^{\prime}(e)+\sum_{e \in F_{1}^{\prime}}\left(1-x^{\prime}(e)\right)$ to simplify the notation. Let $\left(S^{\prime}, F_{0}^{\prime}, F_{1}^{\prime}\right)$ be a minimizer of $g\left(F_{0}^{\prime}, F_{1}^{\prime}\right)$ subject to $\left(F_{0}^{\prime}, F_{1}^{\prime}\right)$ is a partition of $\delta_{G^{\prime}}\left(S^{\prime}\right)$ and $b^{\prime}\left(S^{\prime}\right)+\left|F_{1}^{\prime}\right|$ is odd. Among minimizers, we choose $\left(S^{\prime}, F_{0}^{\prime}, F_{1}^{\prime}\right)$ so that $F_{0}^{\prime} \cup F_{1}^{\prime}$ is inclusion-wise minimal. To derive a contradiction, assume that $g\left(F_{0}^{\prime}, F_{1}^{\prime}\right)<1$. We can show the following claim by a case analysis (see 21] for a proof).

Claim. Let $T \in \mathcal{T}$ be a triangle as shown in Figure 4 and denote $\hat{F}_{0}=F_{0}^{\prime} \cap E_{T}^{\prime}$ and $\hat{F}_{1}=F_{1}^{\prime} \cap E_{T}^{\prime}$. Then, we obtain the following.

(i) If $v_{1}, v_{2}, v_{3} \notin S^{\prime}$, then $r_{T} \notin S^{\prime}$.

(ii) If $v_{1}, v_{2}, v_{3} \in S^{\prime}$, then $r_{T} \in S^{\prime}$.

(iii) If $v_{1} \in S^{\prime}, v_{2}, v_{3} \notin S^{\prime}$, and $\left|\hat{F}_{1}\right|$ is even, then $g\left(\hat{F}_{0}, \hat{F}_{1}\right)=x^{\prime}\left(e_{1}\right)=x(\alpha)+$ $x(\gamma)-2 y_{\alpha \gamma}$.

(iv) If $v_{1} \in S^{\prime}, v_{2}, v_{3} \notin S^{\prime}$, and $\left|\hat{F}_{1}\right|$ is odd, then $g\left(\hat{F}_{0}, \hat{F}_{1}\right)=1-x^{\prime}\left(e_{1}\right)=$ $y_{\emptyset}+y_{\beta}+y_{\alpha \gamma}$.

Note that each $T \in \mathcal{T}$ satisfies exactly one of (i)-(iv) by changing the labels of $v_{1}, v_{2}$, and $v_{3}$ if necessary. In what follows, we construct $\left(S, F_{0}, F_{1}\right) \in \mathcal{F}$ for which $(x, y)$ violates $(9)$. We initialize $\left(S, F_{0}, F_{1}\right)$ as

$$
S=S^{\prime} \cap V, \quad F_{0}=F_{0}^{\prime} \cap E, \quad F_{1}=F_{1}^{\prime} \cap E,
$$

and apply the following procedures for each triangle $T \in \mathcal{T}$.

- If $T$ satisfies the condition (i) or (ii), then we do nothing.

- If $T$ satisfies the condition (iii), then we add $\alpha$ and $\gamma$ to $F_{0}$.

- If $T$ satisfies the condition (iv), then we add $\alpha$ to $F_{0}$ and add $\gamma$ to $F_{1}$.

Then, we obtain that $\left(F_{0}, F_{1}\right)$ is a partition of $\delta_{G}(S), b(S)+\left|F_{1}\right| \equiv b^{\prime}\left(S^{\prime}\right)+\left|F_{1}^{\prime}\right| \equiv$ $1(\bmod 2)$, and

$$
\sum_{e \in F_{0}} x(e)+\sum_{e \in F_{1}}(1-x(e))-\sum_{T \in \mathcal{T}_{S}} 2 q^{*}(T)=\sum_{e \in F_{0}^{\prime}} x^{\prime}(e)+\sum_{e \in F_{1}^{\prime}}\left(1-x^{\prime}(e)\right)<1
$$

by the above claim. This shows that $(x, y)$ violates $(9)$ for $\left(S, F_{0}, F_{1}\right) \in \mathcal{F}$, which completes the proof of the "if" part. 